\title{
Analysis of whole genome-wide microRNA transcriptome profiling in invasive pituitary adenomas and non-invasive pituitary adenomas
}

Chao Zhang ${ }^{1 \dagger}$, Yuan Qian ${ }^{2,3+}$, Yisheng Qiao ${ }^{1}$, Yao Li ${ }^{1}$, Wei Wang ${ }^{1}$, Junjun $\mathrm{Li}^{1}$ and Xingli Deng ${ }^{1 *}$

\begin{abstract}
Background: Dysregulation of microRNAs (miRNAs) plays a critical role during the occurrence and progress of pituitary adenomas (PAs). However, the roles of miRNAs in the invasiveness of PA are poorly understood. This study aims to more comprehensively and specific define the relationship between altered miRNA and PA invasion.

Methods: The differential expression of miRNAs (DEMs) between invasive PAs (IPAs) and non-invasive PAs (NPAs) was explored by RNA sequencing and which functions were analyzed by gene ontology (GO) as well as Kyoto Encyclopedia of Genes and Genomes (KEGG). The miRNA-mRNA network was predicted with bioinformatics.

Results: We identified 31 upregulated miRNAs and 24 downregulated miRNAs in IPAs compared with NPAs. GO analysis and KEGG pathway analysis showed the DEMs were mainly associated with cell proliferation and cell cycle pathway. In addition, on the count of predicted miRNA-mRNA network, two hub miRNAs were identified.

Conclusions: Our results demonstrate the miRNA-mRNA network in detail, which suggest that miRNA may be a promising target in diagnosis and therapy for IPAs.
\end{abstract}

Keywords: Pituitary adenoma, Invasive, MicroRNA, Transcriptome

\section{Background}

PA is one of the most common intracranial tumors with an incidence of $10-15 \%$ [1]. Generally, PAs are considered benign, but some of them are invasive, which invade the adjacent structures such as sphenoid sinus, cavernous sinus, and diaphragma sellae [2-4]. Therefore, IPAs are not only more difficult to complete surgical resection, but also more likely to recurrence after surgery.

MiRNAs are single-strand non-coding RNAs of approximately 19-23 nt, which regulate gene expression at the post-transcriptional level [5, 6], and they can also act as tumor suppressor genes or oncogenes in various tumors [7]. For instance, miR-193b exerts tumor suppressive effects in human acute myeloid leukemia by inducing

\footnotetext{
* Correspondence: dxlkmmu@163.com

${ }^{\dagger}$ Chao Zhang and Yuan Qian contributed equally to this work.

${ }^{1}$ Department of Neurological Surgery, 1st Affiliated Hospital of Kunming

Medical University, Kunming, China

Full list of author information is available at the end of the article
}

tumor cell apoptosis and G1/S arrest [8], while miR-2103 p plays an oncogene role in prostate cancer by promoting cancer cell epithelial-mesenchymal transition and bone metastasis via NF-kB signaling pathway [9]. Altered expression of many miRNAs has been described in PAs, and specific miRNA signatures are related to clinical and therapeutic characteristics of the tumors [10]. However, comprehensive and specific researches of relationships between miRNAs and invasiveness of PAs are still rare.

In order to better understand the mechanism of invasiveness in PAs, it is necessary to clarify the miRNA regulatory network in IPAs. In this study, we detected DEMs in IPAs and NPAs by RNA sequencing, and established the co-expression network contain miRNAs and predicted target genes by Cytoscape. In addition, the expression of the most upregulated miR-665 and the most downregulated miR-149-3p in IPAs was screen out. Moreover, we explained the potential functions of

(c) The Author(s). 2019 Open Access This article is distributed under the terms of the Creative Commons Attribution 4.0 International License (http://creativecommons.org/licenses/by/4.0/), which permits unrestricted use, distribution, and reproduction in any medium, provided you give appropriate credit to the original author(s) and the source, provide a link to the Creative Commons license, and indicate if changes were made. The Creative Commons Public Domain Dedication waiver (http://creativecommons.org/publicdomain/zero/1.0/) applies to the data made available in this article, unless otherwise stated. 
the two key miRNAs in invasive behavior of PAs by GO analysis and KEGG pathway analysis.

\section{Methods}

\section{Patients and samples}

Seven tumor samples were obtained from patients with PAs who underwent operation at the Department of Neurosurgery, 1st Affiliated Hospital of Kunming Medical University for identification of miRNAs by highthroughput sequencing. None of these patients has been received radiotherapy or chemotherapy before surgery. Tumor samples were divided into 2 groups according to invasive behavior proved by surgical findings and pathology: IPA and NPA. All patients were informed according to inform consent approved by the Ethics Boardof the 1st Affiliated Hospital of Kunming Medical University. Immediately following separation, the fresh tumor samples were placed in sterile, RNase-free $2.0-\mathrm{mL}$ cryotubes. Then, samples were soaked in Trizol and stored at $-80^{\circ} \mathrm{C}$ for following analysis.

\section{RNA isolation, library preparation, and sequencing analysis}

Total RNA was extracted from tissue samples by Trizol regent. The integrity of total RNA was detected by agarose electrophoresis and which was quantified by NanoDrop spectrophotometer. Then, the sequencing sample library was constructed by the following steps: ribosomal RNA removal, fragmentation, first-strand complementary DNA (cDNA) synthesis, second-strand cDNA synthesis, terminal repair 3 ' terminal addition, ligation, and enrichment. The libraries were sequence on an Illumina Hiseq 2500/2000 platform.

\section{MiRNA expression analysis}

MiRNA expression levels were estimated by the TPM (transcript per million) through the following criteria: Normalized expression $=$ mapped readcount $/$ Total reads $\times 10^{6}[11]$. All data were analyzed using the DESeq2 $\mathrm{R}$ package (1.8.3). $\log 2 \mathrm{FC}>1$ and $p<0.05$ were considered as the cutoff values for DEMs screening [12].

\section{MiRNA-mRNA network construction}

Based upon results of DEM analysis and target gene prediction, the miRNA-mRNA pairs were extracted to construct the miRNA-mRNA regulatory network. Then, the regulatory network was visualized using Cytoscape_v3.5.1.

\section{Target gene prediction, gene ontology, and pathway enrichment analysis}

Target genes of the DEMs were predicted using major online tools, including miRanda (http://miranda.org.uk/), PITA (http://genie.weizmann.ac.il/pubs/mir07/mir07_data.html), and RNAhybrid (https://bibiserv.cebitec.uni-bielefeld.de/rnahybrid/) [13]. In order to analyze the main functions of the predicted target genes for the DEMs, we performed GO analysis [14]. Moreover, KEGG [15] pathway enrichment analysis was used to find out the significant pathway of predicted target genes for the DEMs. A Go term or KEGG pathway with FDR $<0.05$ was considered statistically significant. Top 10 enriched GO terms and pathways of DEMs were ranked by enrichment score $(-\log 10(p$ value $))$.

\section{Results}

\section{Quality assessment of sequencing data}

The results showed the patterns of gene expression among the samples were similar, and the Pearson correlation between samples is similar in the two groups (Fig. $1 a, b)$. In view of the fact that the length of human miRNAs is generally 19-23 nt, the analysis of the length of reads of each sample shows that 19-23 nt accounts for a higher proportion of each of 2 groups (Fig. 1c, d). And reads of the length region were included in the analysis range. In addition, by comparing and analyzing the error rate among all samples, we found that the read error rate at $19-23 \mathrm{nt}$ was far less than $0.05 \%$ (Fig. 1e, f). All above results indicate the sequencing data is reliable for further bioinformation analysis.

\section{Apportionment and annotation of DEMs}

After summarizing and classifying the sequence reads into different RNA categories, such as miRNA, sn/ snoRNA, tRNA, and rRNAs, the pie chart is drawn to annotate and classify the total reads. The proportion of known miRNAs $(\mathrm{NIN}=63.77 \%, \quad \mathrm{INV}=56.92 \%) \quad$ and newly discovered miRNAs $(\mathrm{NIN}=0.04 \%, \mathrm{INV}=0.02 \%$ ) can be obtained (Fig. 2a, b).

Next, Venn diagram of the DEMs in the two groups was drawn by FunRich3.1.3. A total of 136 DEMs were found in IPA, and 187 DEMs were found in NPA (Fig. 2c).

And then, the volcano map was used to infer the overall distribution of miRNAs. $\square \log 2 \mathrm{FC}>1$ and $p<0.05$ were used as thresholds to screen the DEMs. A total of 31 significantly upregulated and 24 significantly downregulated miRNAs were screened out (Fig. 2d).

At last, these 55 DEMs were used to construct a hierarchical clustering analysis map (Fig. 2e).

\section{GO and KEGG pathway analysis}

In order to explore the functions of DEMs, target genes of these miRNAs were predicted by miRanda, PITA, and RNAhybrid. GO analysis and KEGG pathway analysis were used to their target pool. As results, the results of the top 10 enriched biological process (BP) terms showed that the target gene of DEMs was associated with cell proliferation, cell cycle, and apoptosis process. The top 10 KEGG pathways showed the DEMs might be 


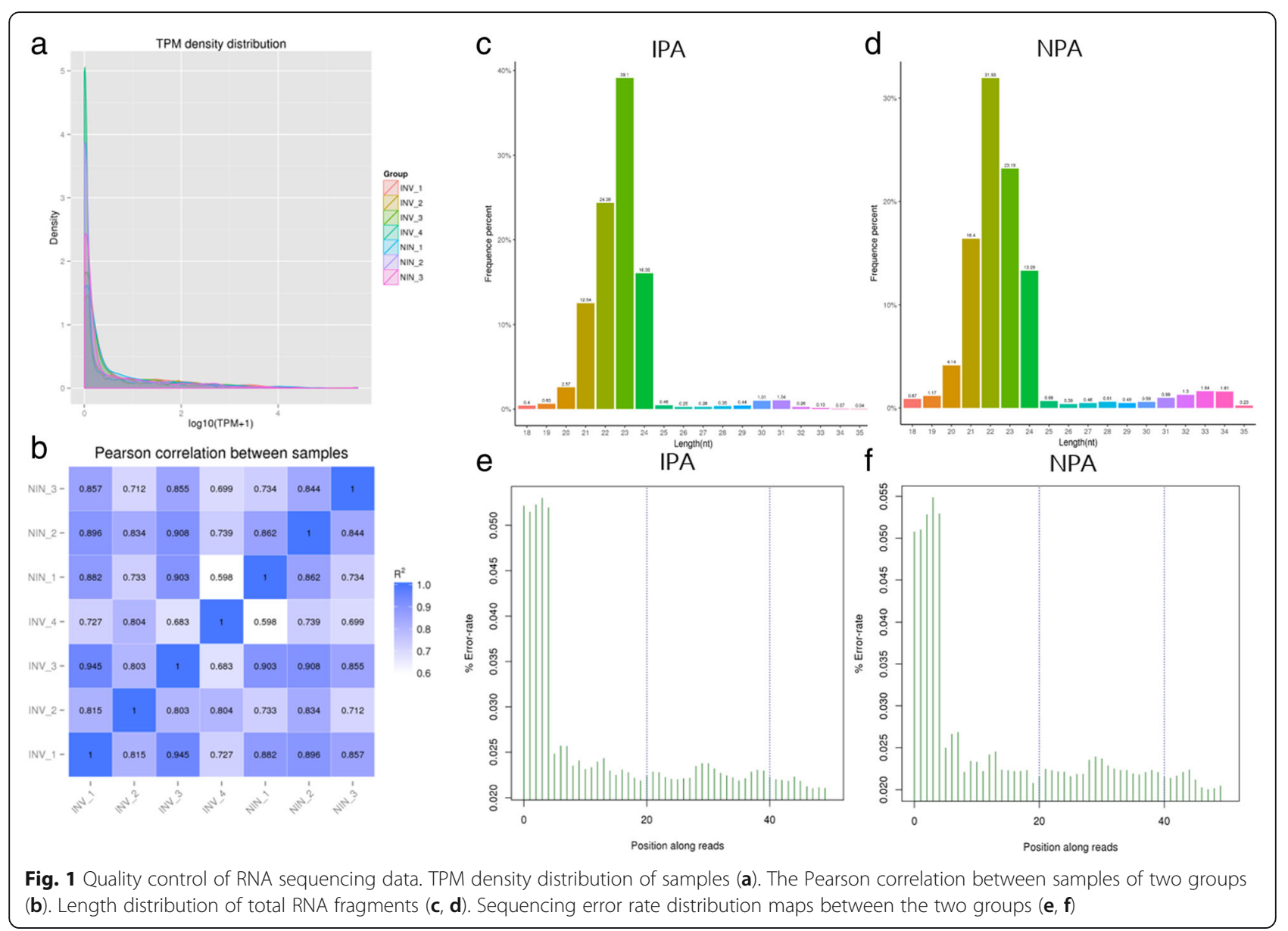

involved in CDK4/6 signaling pathway, PI3K-Akt signaling pathway, and apoptosis pathways (Fig. 3).

\section{MiRNA-mRNA network}

Based on the DEMs and the prediction of target genes. The miRNA-mRNA network was generated by Cytoscape(v3.5.1). As we all know, hub nodes play important roles in biological networks. According to the degree of DEMs calculated by Cytoscape, a total of 12 miRNAs with higher values were identified (Table 1). Then the miRNA-mRNA subnetwork was generated, which included 5 upregulated miRNAs, 7 downregulated miRNAs, and 258 predicted target genes (Fig. 4).

\section{Discussion}

Although PAs are classified into multiple subgroups based on the histological structure, pathological types, and hormone secretion $[16,17]$, the definition of clinically IPAs currently differs in the literature. In according to the 4th edition of the WHO classification of endocrine tumors published in 2017, the invasiveness of PAs can be evaluated through the tumor proliferative capacity by mitotic count and Ki-67 index [18], indicating that the tumor proliferation capacity is closely related to invasion of PAs.

MiRNAs, mediate post-transcriptional regulation, play an important role in epigenetic regulation. Its precursor is cleavage by Dicer enzyme, and then combined with AGO protein and other components to form RISC (RNA immuno-silencing complex) to play a key role in silencing or degradation of target mRNA [19, 20]. Some researchers have reported the regulating functions of miRNAs in PAs [21]. Upregulated miR-34a can significantly inhibit the proliferation of PA cells $\mathrm{GH} 4 \mathrm{C} 1$ and promote apoptosis by regulating SOX7 [22]. Overexpression of miR-16 inhibits the proliferation of PA cells HP75 and promotes apoptosis by targeting HMGA2 expression [23]. However, the comprehensive and specific effects of miRNAs in PA invasion behavior are still rarely reported [24]. Thus, we recognized 55DEMs in IPAs by RNA sequencing analysis. Then, according to Cytoscape calculation, a miRNAmRNA co-expression network including 5 upregulated miRNAs, 7 downregulated miRNAs, and 258 predicted target genes was generated in IPAs.

To further understand the potential function of miRNAs, GO and KEEG pathway analysis were applied for 


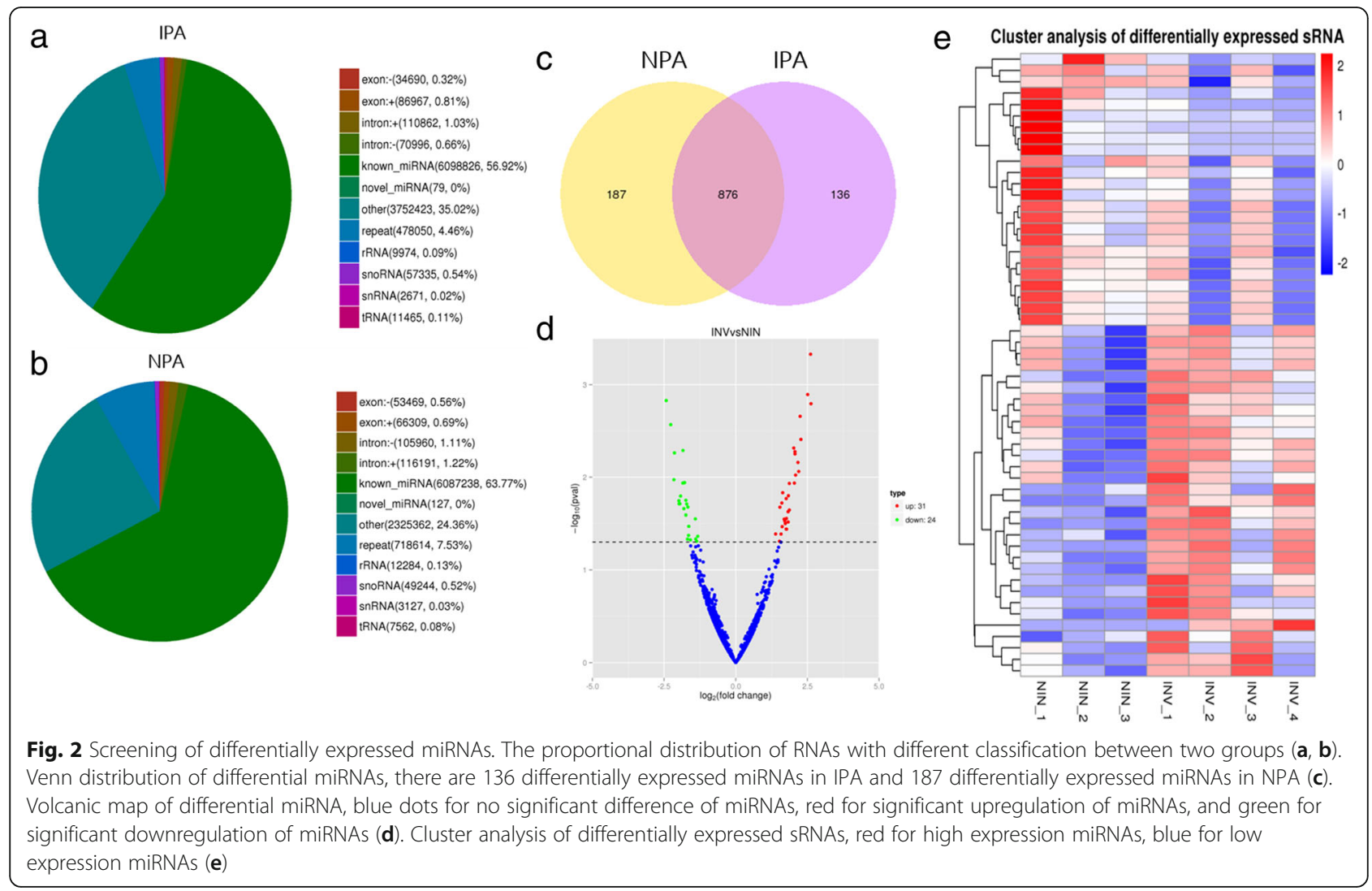

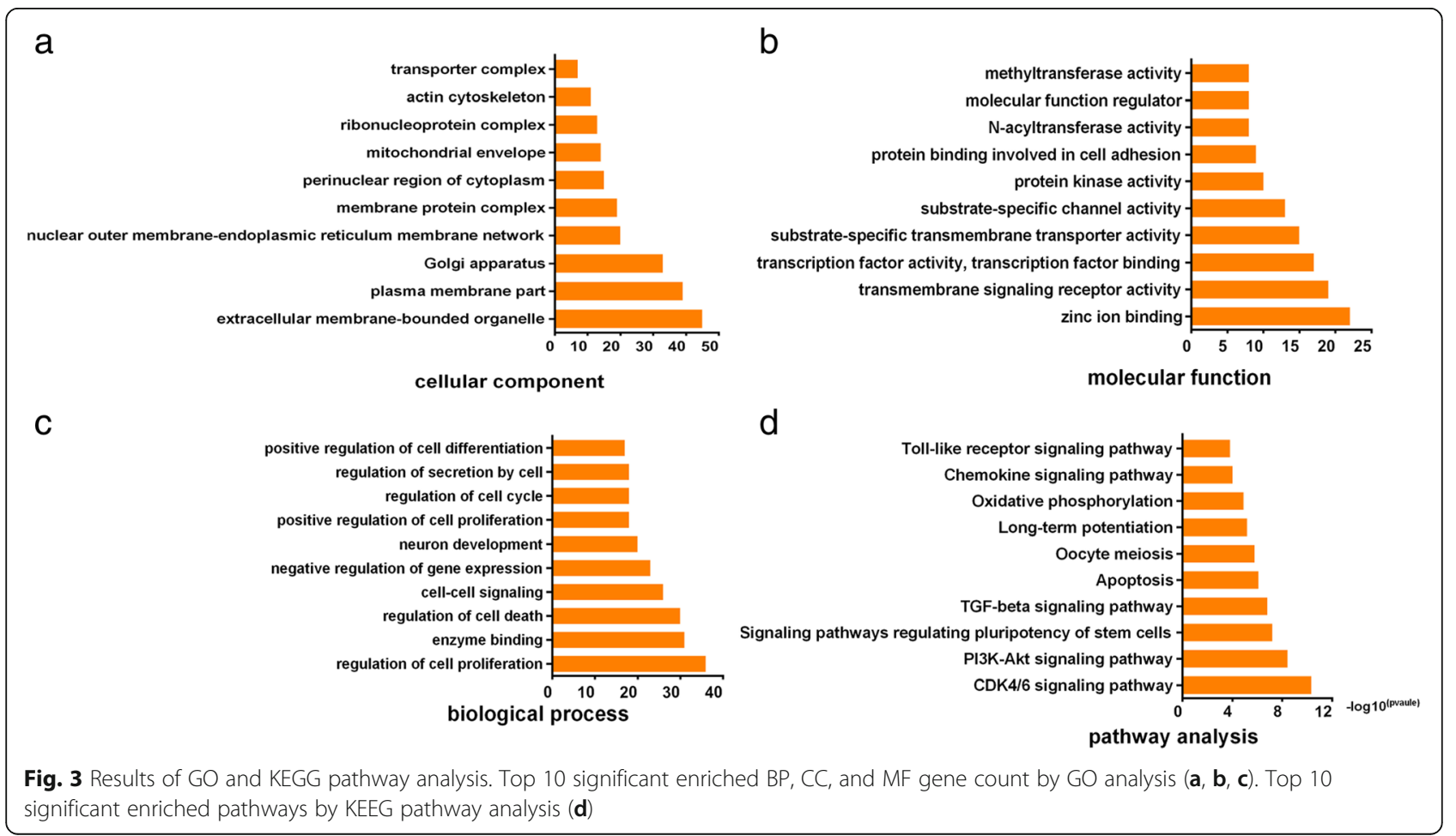


Table 1 The node scores of miRNAs calculated by Cytoscape. The expression profiles of the upregulation and downregulation of miRNA

\begin{tabular}{llllll}
\hline sRNA & Degree & INV_readcount & NIV_readcount & log2FoleChange & $p$ value \\
\hline has-miR-149-3p & 45 & 0.162776612 & 5.71850257 & -2.1576 & 0.0010662 \\
has-miR-502-5p & 6 & 255.3260392 & 2064.122332 & -2.1387 & 0.005467 \\
has-miR-660-3p & 8 & 5.187907073 & 2664.122332 & -1.9248 & 0.016021 \\
has-miR-3615 & 7 & 98.08904874 & 617.924121 & -1.8092 & 0.021926 \\
has-miR-532-3p & 13 & 159.302162 & 1183.189253 & -1.7396 & 0.017793 \\
has-miR-7704 & 32 & 6.42917649 & 31.78715328 & -1.7119 & 0.019517 \\
has-miR-146b-3p & 5 & 96.2448944 & 481.269913 & -1.6392 & 0.033807 \\
has-miR-665 & 79 & 4.742131447 & 0.001235135 & 1.7575 & 0.036171 \\
has-miR-323a-5p & 14 & 4554.136246 & 870.3564614 & 1.7582 & 0.017047 \\
has-miR-134-3p & 12 & 20.80177896 & 2.293937139 & 1.8693 & 0.022669 \\
has-miR-329-5p & 7 & 963.6719203 & 135.5546582 & 2.065 & 0.005295 \\
has-miR-770-5p & 30 & 18.03698596 & 0.409414746 & 2.625 & 0.001605 \\
\hline
\end{tabular}

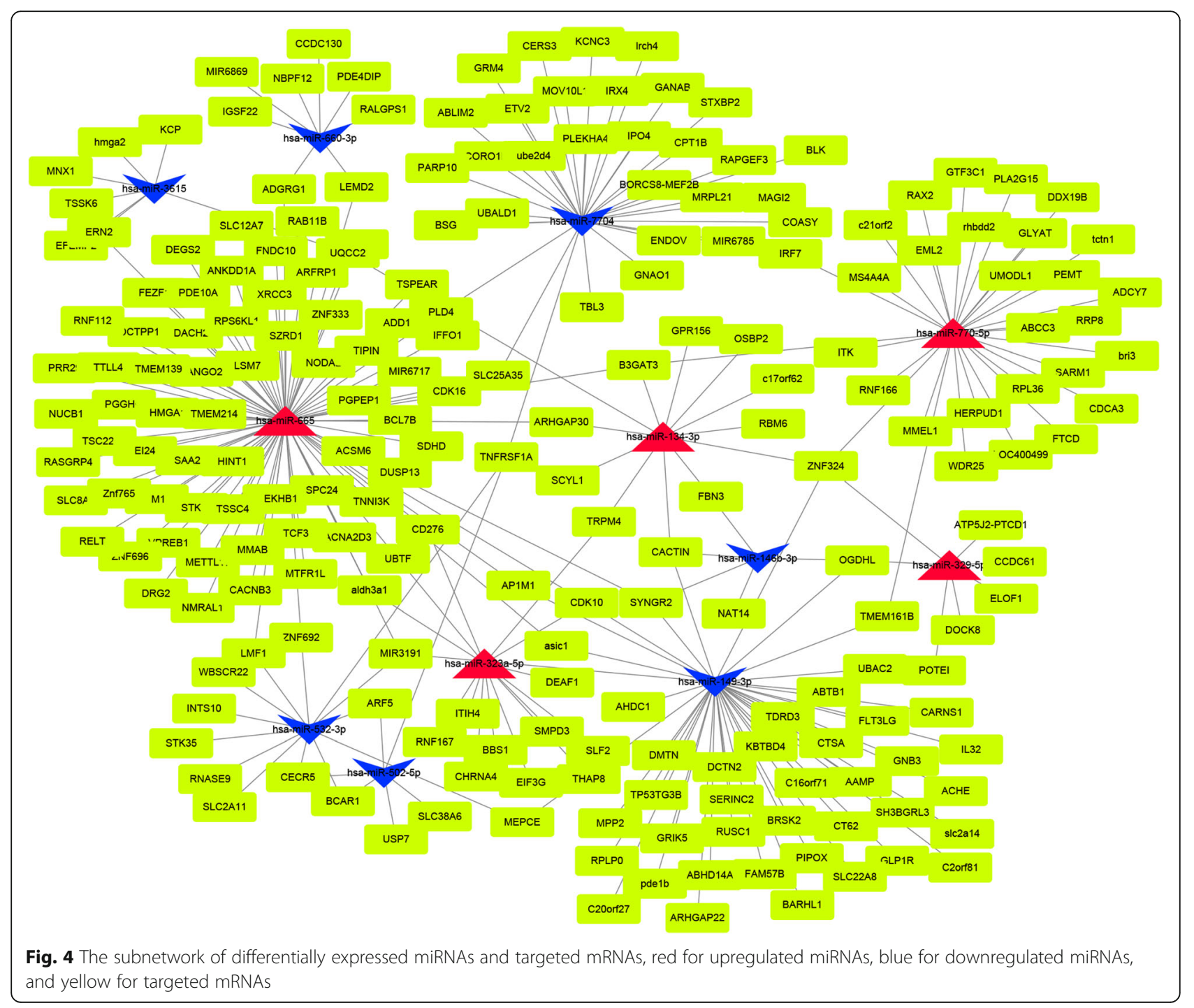


analyzing their possible biological functions and the signaling pathways in IPAs. The outcome of GO analysis showed that the target genes for the DEMs in IPAs were enriched for genes associated with cell proliferation, cell cycle, and apoptosis process, which is consistent with the active proliferation characteristics of IPA cells. Meanwhile, KEEG pathway analysis showed that the DEMs might be involved in CDK4/6 signaling pathway, PI3K-Akt signaling pathway, and apoptosis pathways. It is noted that the enriched terms of target genes for the DEMs from KEEG analysis were consistent with GO analysis results, and they are all closely related to cell proliferation. As mentioned above, tumor proliferation capacity is the most important feature of IPAs.

Additionally, the miRNA-mRNA network was constructed and the outcome showed that some hub miRNAs play important roles in IPAs. The hub miRNAs were identified by the degree of DEMs calculated by Cytoscape. Intriguingly, we found that these miRNAs have similar biological functions. For instance, hsa-miR665 , the most upregulated node in IPAs, can promote tumor cell proliferation or cell cycle progression in hepatocellular carcinoma [25]. In contrast, it was found that overexpression of has-miR-149-3p, the most downregulated node in IPAs, can inhibit the proliferation and invasion of tumor cells in bladder cancer and renal epithelial cell carcinoma [26].

\section{Conclusions}

In this study, we identified the DEMs related to the invasive behavior of PAs. Further study is needed to confirm the exact relationship between DEMs and invasive behavior of PAs and clarify the molecular mechanism of miRNA affecting the invasion of PAs.

\section{Abbreviations}

BP: Biological process; DEM: Differential expression miRNA; GO: Gene ontology; IPA: Invasive pituitary adenoma; KEEG: Encyclopedia of Genes and Genomes; MiRNA: MicroRNA; NPA: Non-invasive pituitary adenoma; PA: Pituitary adenoma

\section{Acknowledgements}

Not applicable

\section{Authors' contributions}

$Y Q$ and $C Z$ contributed to data analysis and writing of the manuscript. $Y Q$, $Y L, W W$, and $J L$ contributed to the execution and critical revisions. XD contributed to conception, design, and final approval of manuscript. All authors read and approved the final manuscript.

\section{Funding}

This study was supported by the Applied Basic Research Foundation of Yunnan Province, the Research Innovation Team of Yunnan Province, the Medical Academic Leader Training Program of Yunnan Province, and the Outstanding Youth Scientists and Technicians Training Program of Yunnan Province.

Availability of data and materials

Please contact author for data requests.
Ethics approval and consent to participate

The study was approved by the Ethics Board of the 1st Affiliated Hospital of Kunming Medical University (NO. 2018NS01).

\section{Consent for publication}

Not applicable

\section{Competing interests}

The authors declare that they have no competing interests.

\section{Author details}

${ }^{1}$ Department of Neurological Surgery, 1st Affiliated Hospital of Kunming Medical University, Kunming, China. ${ }^{2}$ Yunnan Key Laboratory of Laboratory Medicine, Yunnan Engineering Technology Center of Digestive disease, 1st Affiliated Hospital of Kunming Medical University, Kunming, China. ${ }^{3}$ Genetic Diagnosis Center, Kunming City Maternal and Child Health Hospital, Kunming, China.

Received: 30 June 2019 Accepted: 30 October 2019

Published online: 02 December 2019

\section{References}

1. Aflorei ED. Epidemiology and etiopathogenesis of pituitary adenomas. J Neuro-Oncol. 2014;117(3):379-94.

2. Knosp E, Steiner E, Kitz K. Pituitary adenomas with invasion of the cavernous sinus space: a magnetic resonance imaging classification compared with surgical findings. Neurosurgery. 1993,33(4):610-617; discussion 617-8.

3. Farrell WE. Molecular pathogenesis of pituitary tumors. Front Neuroendocrinol. 2000;21(3):174-98.

4. Yang Q. Molecular network basis of invasive pituitary adenoma: a review. Front Endocrinol. 2019;10:7.

5. Berindan-Neagoe I, Monroig Pdel C, Pasculli B. MicroRNAome genome: a treasure for cancer diagnosis and therapy. CA Cancer J Clin. 2014; 64(5):311-36

6. Treiber T, Treiber N. Regulation of microRNA biogenesis and its crosstalk with other cellular pathways. Nat Rev Mol Cell Biol. 2019;20(1):5-20.

7. Rupaimoole R, Slack FJ. MicroRNA therapeutics: towards a new era for the management of cancer and other diseases. Nat Rev Drug Discov. 2017;16(3):1-19.

8. Bhayadia R, Krowiorz K, Haetscher N, et al. Endogenous tumor suppressor microRNA-193b: therapeutic and prognostic value in acute myeloid leukemia. J Clin Oncol. 2018;36(10):1007-17.

9. Ren D, Yang Q, Dai Y, et al. Oncogenic miR-210-3p promotes prostate cancer cell EMT and bone metastasis via NF-KB signaling pathway. Mol Cancer. 2017:16(1):117

10. Gentilin E, Degli Uberti E, Zatelli MC. Strategies to use microRNAs as therapeutic targets. Best Pract Res Clin Endocrinol Metab. 2016;30(5):629-39.

11. Zhou L, Chen J, Li Z, et al. Integrated profiling of microRNAs and mRNAs: microRNAs located on Xq273 associate with clear cell renal cell carcinoma. PloS One. 2010;5(12):e15224

12. Lai $Y$. A statistical method for the conservative adjustment of false discovery rate (q-value). BMC Bioinformatics. 2017;18(Suppl 3):69.

13. Wu HJ, Ma YK, Chen T, et al. PsRobot: a web-based plant small RNA metaanalysis toolbox. Nuc Acids Res. 2012;40(Web server issue):W22-8.

14. Chen CM, Lu YL, Sio CP, et al. Gene ontology based housekeeping gene selection for RNA-seq normalization. Methods (San Diego, Calif.), 2014, 67(3): 354-363.

15. Mao X, Cai T, Olyarchuk JG. Automated genome annotation and pathway identification using the KEGG Orthology $(\mathrm{KO})$ as a controlled vocabulary. Bioinformatics (Oxford, England). 2005;21(19):3787-93.

16. Micko AS, Wöhrer A, Wolfsberger S. Invasion of the cavernous sinus space in pituitary adenomas: endoscopic verification and its correlation with an MRIbased classification. J Neurosurg. 2015;122(4):803-11.

17. Kalinin PL, Sharipov OI, Pronin IN, et al. Endoscopic transsphenoidal resection of pituitary adenomas invading the cavernous sinus. Zh Vopr Neirokhir Im N N Burdenko. 2016;80(4):63-74.

18. Lopes MBS. The 2017 World Health Organization classification of tumors of the pituitary gland: a summary. Acta Neuropathol. 2017;134(4):521-35.

19. Shukla GC, Singh J. MicroRNAs: processing, maturation, target recognition and regulatory functions. Mol Cell Pharmacol. 2011;3(3):83-92. 
20. Meister G, Landthaler M, Patkaniowska A, et al. Human Argonaute2 mediates RNA cleavage targeted by miRNAs and siRNAs. Mol Cell. 2004; 15(2):185-97.

21. Németh K, Darvasi O, Szücs N, et al. The role of miRNAs in the pathogenesis of pituitary adenomas. Orvosi Hetilap. 2018;159(7):252-9.

22. Yang Z, Zhang T, Wang Q. Overexpression of microRNA-34a attenuates proliferation and induces apoptosis in pituitary adenoma cells via SOX7. Mol Ther Oncol. 2018;10:40-7.

23. Niu Y, Zhou H, Liu Y, et al. miR-16 regulates proliferation and apoptosis of pituitary adenoma cells by inhibiting HMGA2. Oncol Lett. 2019;17(2):2491-7.

24. Feng $Y$, Mao ZG, Wang $X$, et al. MicroRNAs and target genes in pituitary adenomas. Horm Metab Res. 2018;50(3):179-92.

25. Hu Y, Yang C, Yang S, et al. miR-665 promotes hepatocellular carcinoma cell migration, invasion, and proliferation by decreasing Hippo signaling through targeting PTPRB. Cell Death Dis. 2018:9(10):954.

26. Zhan Y, Chen Z, Li Y, et al. Long non-coding RNA DANCR promotes malignant phenotypes of bladder cancer cells by modulating the miR-149/ MSI2 axis as a ceRNA. J Exp Clin Cancer Res. 2018;37(1):273.

Ready to submit your research? Choose BMC and benefit from:

- fast, convenient online submission

- thorough peer review by experienced researchers in your field

- rapid publication on acceptance

- support for research data, including large and complex data types

- gold Open Access which fosters wider collaboration and increased citations

- maximum visibility for your research: over $100 \mathrm{M}$ website views per year

At $\mathrm{BMC}$, research is always in progress.

Learn more biomedcentral.com/submissions 